\title{
Seasonal Variation in Readmission Risk for Patients Hospitalized with Cardiopulmonary Conditions
}

\author{
Saul Blecker, MD, $M H S^{7}$, Ji Young Kwon, MPH², Jeph Herrin, $P h D^{3}$, Jacqueline N. Grady, $M S^{2}$, and \\ Leora I. Horwitz, MD, MHS
}

'Division of Healthcare Delivery Science, Department of Population Health, NYU School of Medicine, New York, NY, USA; ${ }^{2}$ Center for Outcomes Research and Evaluation, Yale-New Haven Hospital, New Haven, CT, USA; ${ }^{3}$ Section of Cardiovascular Medicine, Yale School of Medicine, New Haven, CT, USA.

J Gen Intern Med 33(5):599-601

DOI: $10.1007 / \mathrm{s} 11606-017-4299-0$

(c) Society of General Internal Medicine 2018

$\mathrm{P}$ revious epidemiologic studies have suggested a seasonal variation in hospitalizations for cardiorespiratory conditions, including heart failure, pneumonia, and chronic obstructive pulmonary disease (COPD), with highest prevalence of admissions in winter months. ${ }^{1-3}$ We recently studied trends in risk of readmission among hospitalized Medicare beneficiaries and observed a seasonal variation in predicted readmission risk for patients hospitalized for cardiorespiratory conditions, but not for patients hospitalized for other reasons. ${ }^{4}$ Specifically, predicted readmission risk for beneficiaries hospitalized for cardiorespiratory conditions was highest in the summer months and lowest in winter months. The purpose of this study was to examine whether seasonal differences in risk were related to variations in reason for admission or due to seasonal variations in risk strata.

\section{METHODS}

We conducted a retrospective study of all cardiorespiratory hospitalizations of Medicare fee-for-service beneficiaries between January 1, 2009, and June 30, 2015. Cardiorespiratory hospitalization was based on principal discharge diagnosis using Agency for Healthcare Research and Quality clinical classification software. ${ }^{5}$ We divided these hospitalizations into four condition groups based on principal discharge diagnosis: heart failure, pneumonia, COPD, and other cardiorespiratory conditions, which included asthma, pulmonary heart disease, bronchitis, and respiratory failure. We examined monthly trends in the number of hospitalizations over time for each condition group.

We then categorized all hospitalizations based on predicted risk of readmission. As previously described, ${ }^{4}$ we used generalized estimating equations to develop a risk prediction

Published online February 20, 2018 model for 30-day unplanned hospital readmission. Variables included in the model were those in the final hospital-wide readmission model ${ }^{5}$ plus additional characteristics including sex, race, dual eligibility status, prior discharges against medical advice, intensive care unit stay, and prior hospitalizations. Based on the model, each hospitalization was categorized as lowest $20 \%$, middle $60 \%$, and highest $20 \%$ of predicted readmission risk. We calculated means and standard deviations of the number of hospitalizations and examined monthly trends in number of hospitalizations for each risk stratum. For the highest and lowest risk strata, we calculated the mean and range of difference in the number of hospitalizations in January and August, the months with the highest and lowest number of hospitalizations, respectively, for years 2009-2014. To simplify comparisons, we divided the totals for the middle $60 \%$ stratum by three and normalized months to 30 days for all analyses.

\section{RESULTS}

Of $8,179,691$ cardiopulmonary hospitalizations, 2,661,837 $(32.5 \%)$ were for heart failure, 2,308,866 (28.2\%) were for pneumonia, 1,722,347 (21.1\%) were for COPD, and $1,486,641(18.2 \%)$ were for other conditions. The mean (SD) number of hospitalizations was $129,083(10,092)$ in January and 83,456 (4515) in August, the months with the highest and lowest number of hospitalizations, respectively. We observed an increase in the number of hospitalizations in winter months for all conditions, with the increase in hospitalizations greatest for patients with pneumonia (Fig. 1).

The overall monthly mean number of hospitalizations was similar for each readmission risk stratum although variances differed, with means (SD) of 20,981 (4705), 20,967 (3343), and 20,966 (2229) for the lowest, middle, and highest risk strata, respectively. We observed seasonal variation in number of hospitalizations for all three risk strata, although the magnitude of variation differed by risk stratum (Fig. 2). The mean difference in hospitalizations 


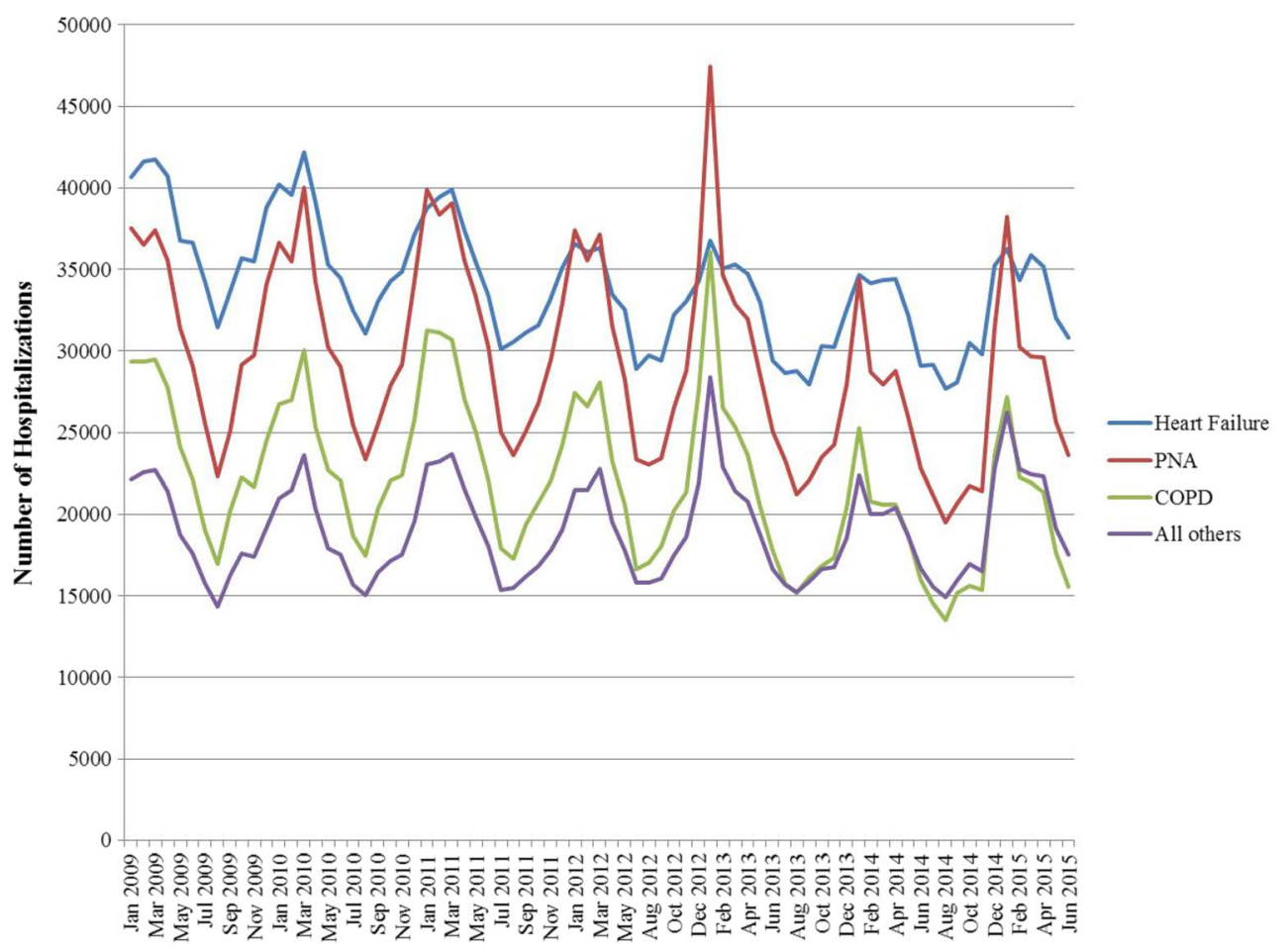

Figure 1 Number of hospitalizations per month for Medicare beneficiaries hospitalized for heart failure, pneumonia, COPD, and other cardiopulmonary conditions.

between January versus August was 4518 (range 34046598 ) for patients in the highest quintile of readmission risk and 13,217 (range 9929-22,431) for patients in the lowest quintile of risk.

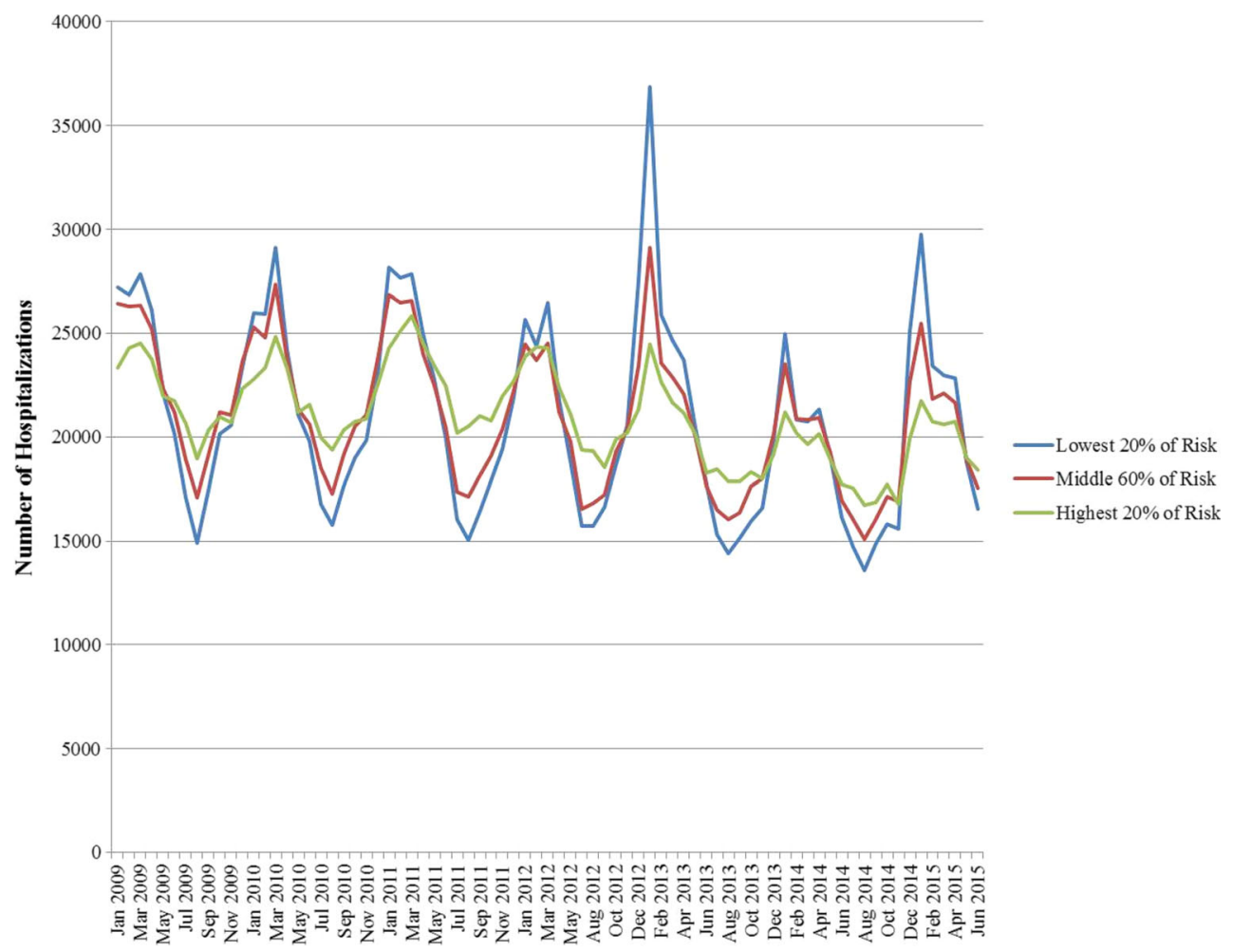

Figure 2 Number of hospitalizations by month for Medicare beneficiaries hospitalized for cardiopulmonary conditions, by strata of predicted risk of readmission. Number of hospitalizations in the middle $60 \%$ of risk was divided by three for comparability. 


\section{DISCUSSION}

We observed significant increases in hospitalizations during winter months for heart failure, pneumonia, COPD, and other cardiopulmonary conditions. Much of the seasonal variation in hospitalizations for patients with cardiopulmonary conditions appears to be driven by a large increase in hospitalizations for low-risk patients in winter months. Conversely, we found fewer excesses of hospitalizations in winter versus summer months for patients with high baseline risk. Study limitations include potential for misclassification of risk due to imperfect model discrimination and a focus on Medicare beneficiaries, so results may not be generalizable to younger patients.

Our findings suggest an opportunity to reduce hospitalizations through preventative measures targeting low-risk patients in the fall season, such as vaccinations or ambulatory visits.

Acknowledgements: This study was supported by the Agency for Healthcare Research and Quality (AHRQ) grant RO1 HSO22882. Dr. Blecker was supported by the AHRQ grant K08HS23683. We thank Shawn Hoke and Jane Padikkala for administrative support.

Corresponding Author: Saul Blecker, MD, MHS; Division of Healthcare Delivery Science, Department of Population Health NYU School of Medicine, New York, NY, USA (e-mail: saul.blecker@nyumc. org).

\section{Compliance with Ethical Standards:}

Conflict of Interest: The authors declare that they do not have a conflict of interest.

\section{REFERENCES}

1. Rabe KF, Fabbri LM, Vogelmeier C, et al. Seasonal distribution of COPD exacerbations in the Prevention of Exacerbations with Tiotropium in COPD trial. Chest 2013;143(3):711-719.

2. Murdoch KM, Mitra B, Lambert S, Erbas B. What is the seasonal distribution of community acquired pneumonia over time? A systematic review. Australas Emerg Nurs J 2014;17(1):30-42.

3. Patel NJ, Nalluri N, Deshmukh A, et al. Seasonal trends of heart failure hospitalizations in the United States: a national perspective from 2000 to 2011. Int J Cardiol 2014;173(3):562-563.

4. Blecker S, Herrin J, Kwon JY, Grady JN, Jones S, Horwitz LI. Effect of hospital readmission reduction on patients at low, medium, and high risk of readmission in the Medicare population. J Hosp Med.

5. Horwitz LI, Partovian C, Lin Z, et al. Development and use of an administrative claims measure for profiling hospital-wide performance on 30-day unplanned readmission. Ann Intern Med 2014;161(10 Suppl):S66-75 